\title{
Cost-Optimal ATCs in Zonal Electricity Markets
}

\author{
Jensen, Tue Vissing; Kazempour, Jalal; Pinson, Pierre
}

Published in:

IEEE Transactions on Power Systems

Link to article, DOI:

10.1109/TPWRS.2017.2786940

Publication date:

2018

Document Version

Peer reviewed version

Link back to DTU Orbit

Citation (APA):

Jensen, T. V., Kazempour, J., \& Pinson, P. (2018). Cost-Optimal ATCs in Zonal Electricity Markets. IEEE Transactions on Power Systems, 33(4), 3624-3633. https://doi.org/10.1109/TPWRS.2017.2786940

\section{General rights}

Copyright and moral rights for the publications made accessible in the public portal are retained by the authors and/or other copyright owners and it is a condition of accessing publications that users recognise and abide by the legal requirements associated with these rights.

- Users may download and print one copy of any publication from the public portal for the purpose of private study or research.

- You may not further distribute the material or use it for any profit-making activity or commercial gain

- You may freely distribute the URL identifying the publication in the public portal

If you believe that this document breaches copyright please contact us providing details, and we will remove access to the work immediately and investigate your claim 


\title{
Cost-Optimal ATCs in Zonal Electricity Markets
}

\author{
Tue Vissing Jensen, Student Member, IEEE, Jalal Kazempour, Member, IEEE, \\ and Pierre Pinson, Senior Member, IEEE
}

\begin{abstract}
In contrast to existing frameworks for Available Transfer Capacity (ATC) determination, we propose to define ATCs in an integrated and data-driven manner, optimizing for expected operational costs of the whole system to derive cost-optimal ATCs. These ATCs are purely financial parameters, separate from the physical ATCs based on security indices only typically used in zonal electricity markets today. Determining cost-optimal ATCs requires viewing ATCs as an endogenous market construct, and leads naturally to the definition of a market entity whose responsibility is to optimize ATCs. The optimization problem which this entity solves is a stochastic bilevel problem, which we decompose to yield a computationally tractable formulation. We show that cost-optimal ATCs depend non-trivially on the underlying network structure, and the problem of finding a set of cost-optimal ATCs is in general non-convex. On a Europeanscale test system, cost-optimal ATCs achieve expected total costs midway between those for non-integrated ATCs and a fully stochastic nodal setup. This benefit comes from qualitatively different ATCs compared to typical definitions, with ATCs which exceed the physical cross-border capacity by a factor of 2 or more, and ATCs which are zero between well-connected areas. Our results indicate that the perceived efficiency gap between zonal and nodal markets may be exagerrated if non-optimal ATCs are used.
\end{abstract}

Index Terms-Zonal Electricity Markets, Market Coupling, Available Transfer Capacity, Bilevel programming

\section{NOMENCLATURE}

A. Sets
$z \in Z$
Set of zones
$n \in N$
Set of nodes
$N_{\mathrm{Sl}} \subseteq N \quad$ Set of slack buses
$g \in G \quad$ Set of generators
$G_{z} \subseteq G \quad$ Set of generators in zone $z$
$G_{n} \subseteq G \quad$ Set of generators at node $n$
$e \in E \quad$ Set of links in zonal network
$z \rightarrow \subseteq E \quad$ Set of links originating from zone $z$
$\rightarrow z \subseteq E$ Set of links ending at zone $z$
$l \in L \quad$ Set of lines in transmission network
$n \rightarrow \subseteq L \quad$ Set of lines originating from node $n$
$\rightarrow n \subseteq L$ Set of lines ending at node $n$
$\omega \in \Omega \quad$ Set of scenarios for renewable production

\section{B. Variables}

Variables indexed by $\omega$ refer to real-time (RT) markets.

$p_{g}^{\mathrm{DA}}\left(p_{g \omega}^{\mathrm{RT}}\right) \quad$ Production of generator $g$ [MW]

$p_{g \omega}^{\uparrow}, p_{g \omega}^{\downarrow} \quad \mathrm{Up} /$ down regulation of generator $g$ [MW]

T. Jensen, J. Kazempour and P. Pinson are with the Technical University of Denmark, 2800 Kgs. Lyngby DK (e-mail: \{tvjens, seykaz, ppin\}@elektro.dtu.dk)

This work is partly funded by ' $5 \mathrm{~s}$ ' - Future Electricity Markets (12132636/DSF).

$\begin{array}{ll}w_{z}^{\mathrm{DA}}\left(w_{n \omega}^{\mathrm{RT}}\right) \text { Scheduled renewable production in zone } z / \\ & \text { node } n \text { [MW] } \\ f_{e}^{\mathrm{DA}}\left(f_{l \omega}^{\mathrm{RT}}\right) & \text { Flow on link } e / \text { line } l[\mathrm{MW}] \\ l_{z}^{\mathrm{s}, \mathrm{DA}}\left(l_{n \omega}^{\mathrm{s}, \mathrm{RT}}\right) & \text { Load shed in zone } z / \text { node } n[\mathrm{MW}] \\ A T C_{e} & \text { Flow limit for link } e[\mathrm{MW}] \\ \theta_{n \omega} & \text { Phase angle in node } n \text { [rad] } \\ \mathrm{Cost}^{\mathrm{DA}} & \text { Total dispatch cost in the day-ahead market [\$] } \\ \Delta \mathrm{Cost}_{\omega}^{\mathrm{RT}} & \text { Change in dispatch cost from real-time opera- } \\ & \text { tion in scenario } \omega[\$] \\ \alpha_{\omega} & \text { Benders' proxy variable [\$] }\end{array}$

C. Parameters

$\begin{array}{ll}P_{g}^{\max } & \text { Capacity of generator } g \text { [MW] } \\ \pi_{g} & \text { Price offer of generator } g \text { [\$/MWh] } \\ \pi_{g}^{\uparrow / \downarrow} & \text { Regulating offer markup of generator } g[\$ / \mathrm{MWh}] \\ W_{z}^{\mathrm{DA}} & \text { Day-ahead renewable offer in zone } z[\mathrm{MW}] \\ \pi^{\mathrm{r}} & \text { Price offer of renewables [\$/MWh] } \\ W_{n \omega}^{\mathrm{RT}} & \text { Realized renewable production at node } n \text { [MW] } \\ L_{z}^{\mathrm{DA}} & \text { Projected load in zone } z[\mathrm{MW}] \\ L_{n}^{\mathrm{RT}} & \text { Realized load at node } n[\mathrm{MW}] \\ F_{l}^{\mathrm{max}} & \text { Physical flow limit on line } l[\mathrm{MW}] \\ B_{l} & \text { Susceptance of line } l[\mathrm{p} . \mathrm{u} .] \\ V O L L & \text { Value of lost load [\$/MWh] } \\ \phi_{\omega} & \text { Probability of scenario } \omega \\ \alpha^{\mathrm{min}} & \text { Lower bound to Benders' proxy variable }[\$]\end{array}$

\section{INTRODUCTION}

A $S$ renewable penetration increases, the cost of compensating for its uncertainty comes to dominate electricity market operation costs [1]. A major challenge in modern electricity market design is how to properly account for this uncertainty to optimally integrate renewable production [2]. This challenge is even greater in a European context, where current practice dictates that day-ahead prices cannot be directly differentiated by their geographical location, but must be the same across pre-defined market areas, i.e., zones, often an entire country [3]. Under this restriction on prices, any method for which the day-ahead price seen by a market participant can depend on its location inside the zone is untenable. Such methods include stochastic market designs in the vein of [4] and [5] with full network representation, which in this context are the ideal benchmark in terms of expected system cost. The primary degree of freedom left to influence the dispatch is to control the maximal commercial exchange between zones, known as the available transfer capacity, or ATC. ${ }^{1}$

\footnotetext{
${ }^{1}$ Depending on context, the same concept has been referred to as Net Transfer Capacity (NTC) or Total Transfer Capacity (TTC). See [6] for a disambiguation.
} 
(R2.1) This leads us to consider a distinction between physical ATCs on the one hand, which describe the capability of the underlying grid to transfer power between two market zones, and financial ATCs on the other hand, which are limitations enforced on the exchanges scheduled between two market zones in the day-ahead market. While these financial ATCs are historically set equal to physical ATCs based on measures of security irrespective of their market use [7], [8], they influence market clearing by limiting the scheduled energy transfer between zones. This interaction has not been extensively explored. One question which has yet to be fully examined is to which extent the chosen ATCs impact market outcomes, and whether market efficiency can be improved by integrating market clearing into the definition of ATCs.

Previous works on the connection between the definition of ATCs and market outcomes have focused on ATCs as a technical construct derived from the underlying network. In [9], ATCs are defined statically depending only on the network topology, as the maximal two-zone transfer which is robust to the intra-zonal injection pattern. In [10], ATCs are defined according to the expected operating point as the maximal deviation in transfer such that the system is robust. Both methods define ATCs exogenously without reference to their use in market clearing. The work of [11] uses grid search to find the ATC which leads to lowest overall cost for a two-node topology. This method does not scale to large grids, and may not achieve global optimality. The optimal zonal pricing method of [12] gets around defining ATCs by clearing a nodally priced market, including constraints to enforce the same price for all nodes in a zone. This approach requires linear marginal cost functions per node, compared to the typical situation of piecewise linear, and requires the announcement of prices for the full nodal system for the system to be at equilibrium.

(R2.1) We propose to separate the notion of physical ATCs, and their use in markets as financial ATCs. Once the functioning of financial ATCs in markets is separated from physical details, they can be viewed as free parameters, which can in turn be optimized to maximize social welfare. We examine a method of ATC determination for a given topology of zones based on a stochastic setup which (i) uses explicit information on the uncertainty of variable renewable production (VRES, here wind and solar), (ii) respects the zonal pricing system currently in use in European markets, (iii) minimizes the expected total cost of operating the system under renewable uncertainty while respecting transmission limits, and (iv) is computationally tractable for large-scale problems. These features are achieved by setting the ATCs between zones to indirectly control the dispatch of generators towards a dispatch that is better suited to deal with the forecast renewable uncertainty. From the perspective of a market participant, the day-ahead market clears in the exact same way as today, only with different ATCs. The proposed method can be brought closer to current practice by including, e.g., minimum ATCs at the cost of sub-optimality.

Compared to [9], our method determines ATCs which optimally account for scenarios of net load situation, and defines ATCs based on their use in day-ahead markets. Next to [10], we treat ATCs as endogenous to our day-ahead market model, and evaluate them under renewable uncertainty. Our results complement the conclusions of [10] by showing that the perceived performance of zonal market depends on the chosen ATCs. In contrast to [11], our method extends to largescale systems, and is guaranteed to find a set of ATCs which attain the minimum global expected cost. Unlike [12], our method allows for nodes to have any convex cost function, and respects the trading schemes used in current European electricity markets, including the information released to each participant.

The rest of this paper is organized as follows. Section II expands on current ATC practice and introduces the assumptions used for our method. Section III gives the method formulation and its decomposed form. Section IV examines two case studies to reveal features of the problem and its outcomes. Finally, Section V concludes on the results obtained.

\section{Preliminaries And Assumptions}

\section{A. ATC Definition: Current Practice}

The appeal of using generation resources more efficiently across borders and integrating more renewables, drives the coupling of the European electricity system [13]. The physical system comprises several thousand buses $n \in N$ and transmission lines $l \in L$ across the continent. By contrast, in current European day-ahead markets, the transmission grid is simplified to interconnections $e \in E$ between tens of market zones $z \in Z$. This simplified zonal view of the transmission system in the day-ahead market stage challenges system operation, as it does not represent the physical nodal transmission system [9], [14]. Reconciling these zonal and nodal descriptions constitutes unique challenges in market design [9]-[11]. Of particular importance is examining how market zones interact, as efficient trade between market zones lowers system operation cost [13].

When clearing day-ahead markets, market operators of each zone can clear bids across links $e$, limited by the corresponding $A T C_{e}$. Efficient market operation requires properly defining the ATCs. Their definition involves a trade-off between efficient trading of energy and system reliability: If ATCs are too restrictive, fewer trades may be cleared, leading to a loss of social welfare (increased system cost). On the other hand, if ATCs are too lax, too much energy may be scheduled to be transmitted across some lines, which may force use of expensive balancing resources or endanger system reliability.

Historically, Transmission System Operators (TSOs) have defined ATCs from a security standpoint; Given the expected system operating points, find the maximal transfer of energy across the link $e, f_{e}$, that will allow the system to operate securely [10]. Several reliability indices have been proposed to quantify the stability and security of the system, such as loss of load probability and energy not supplied, analyzed under contingencies [7], [8]. Though European markets have in recent years moved from ATC-based day-ahead market coupling to flow-based market coupling [6], the security-based view of market coupling is unchanged. Thus, in current practice ATCs are fundamentally taken to be a technical construct; 


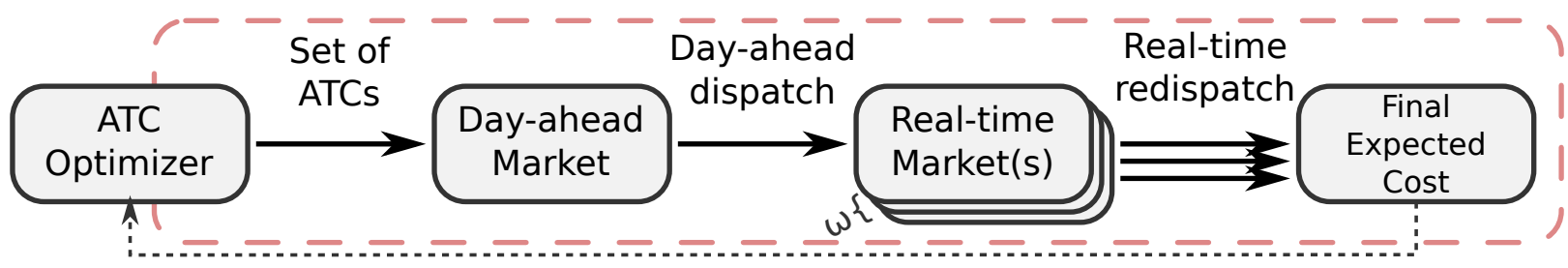

Fig. 1. Functioning of a zonal market structure. Each arrow indicates the outcome of a step, which is input to the following step. The real-time market(s) clear depending on the realized scenario $\omega$. The dashed box indicates the problem of the ATC optimizer.

they represent the physical capabilities of the underlying grid without reference to their use in market clearing and the resulting financial impacts.

However, ATCs are fundamentally a financial concept: they inform market participants how much trade between zones is beneficial to the system as a whole. From this perspective, ATCs are not tightly coupled to the physical grid; so long as the day-ahead trades carried out using ATCs result in a dispatch which allows resolving transmission limit violations in real-time, TSOs should not care what their specific values are. This view suggests that by decoupling ATC definition from the physical grid to some extent, it may be possible for the European system to operate at lower overall cost by optimizing ATCs, a suggestion supported by our findings in this paper. We refer to the optimized ATCs as cost-optimal, opposed to the commonly used non-integrated ATCs. The following subsection builds the machinery needed to define cost-optimal ATCs.

\section{B. ATCs as Degrees of Freedom}

A given set $\left\{A T C_{e}\right\}_{e \in E}$ of ATCs determine final costs through day-ahead market trading decisions and redispatch needs, as illustrated in Fig. 1. In order to lower the final expected cost (system cost in day-ahead, i.e., $\operatorname{Cost}^{\mathrm{DA}}$, plus the mean cost of balancing actions, i.e., $\sum_{\omega} \phi_{\omega} \Delta \operatorname{Cost}_{\omega}^{\mathrm{RT}}$ where $\omega$ is an index for scenarios representing a realization of uncertainty with forecasted probability $\phi_{\omega}$ ), one can consider this diagram as a feedback loop; for any proposed $\left\{A T C_{e}\right\}_{e \in E}$, the final expected cost changes. If the purpose of the market is to minimize final expected cost, this feedback loop should be used to determine ATCs.

This endogenous approach defines the ATC optimizer's problem: Given a belief about day-ahead offers, balancing offers and possible net load scenarios, find $\left\{A T C_{e}\right\}_{e \in E}$ which leads to the lowest expected cost of operation. The ATC optimizer is a market entity who acts as the leader in a Stackelberg game, anticipating the day-ahead market and real-time market(s) acting as sequential followers. Stackelberg games are naturally formulated as bilevel optimization problems [15], with the upper level representing the ATC optimizer's (leader's) problem of defining a set of ATCs, and the lower-level problems (followers) representing the dayahead market clearing and the real-time balancing action, respectively. This bilevel structure is necessary to model the myopic nature of the day-ahead market clearing with respect to the underlying transmission grid. (R2.3) That is, the day-ahead market does not represent real-time operational constraints, including grid constraints.

Formally, the ATC optimizer's problem is written as

$$
\begin{aligned}
& \min _{A T C_{e}} \operatorname{Cost}^{\mathrm{DA}}+\sum_{\omega} \phi_{\omega} \Delta \operatorname{Cost}_{\omega}^{\mathrm{RT}} \\
& \text { s.t. } A T C_{e} \geq 0 \\
& \forall e \in E \\
& \mathrm{Cost}^{\mathrm{DA}}, p^{\mathrm{DA}} \in\left(2 \mid A T C_{e}\right) \\
& \Delta \operatorname{Cost}_{\omega}^{\mathrm{RT}} \in\left(3 \mid p^{\mathrm{DA}}\right)_{\omega} \quad \forall \omega \in \Omega
\end{aligned}
$$

where minimizing final expected cost (1a) determines the optimal non-negative ATCs (1b). Anticipated system costs and day-ahead dispatch (1c) (which depend on the ATCs as indicated by the conditional symbol '|'), and balancing costs in real-time per scenario $\omega$ (1d) (which depend on the day-ahead dispath) are given by their respective lower-level problems. The ATC optimizer must solve (1) for every time period under consideration. (R2.3) The formulation of this problem ensures that the day-ahead and real-time problems are cleared sequentially as in Fig. 1.

The definition of ATCs in (1) involves a trade-off between day-ahead and real-time costs. If ATCs are very high, dayahead cost will be low, while costs in real-time may be high due to significant needs for re-dispatch. On the other hand, if ATCs are very low, day-ahead cost will increase dramatically, while real-time costs will not decrease. An optimal set of ATCs balances these two costs.

We do not propose that European market operators rush out to employ ATC optimizers, but rather view the definition of such a market position as an interesting theoretical construct: The set of ATCs which the ATC optimizer finds yield a lower bound on the cost of operating the zonal market, i.e. it is a fundamental limitation on zonal market clearing as practiced in Europe today. The approach considered here can then be used to assess the efficiency of a zonal market: The closer a zonal market's cost is to the ATC optimizer's optimum, the better the market functions.

\section{Modeling Assumptions and Problem Structure}

In order to formulate this problem in a tractable manner, assumptions on the associated market clearings are necessary.

The day-ahead market is assumed to clear in a fully coordinated fashion, where each zone is assumed to be an energy exchange which allows partial acceptance of offers. Renewables are assumed to offer their mean into the dayahead market at zero price $\left(\pi_{r}=\$ 0 / \mathrm{MW} \mathrm{h}\right)$ and are the only source of uncertainty in the system. The ATC optimizer uses a 
forecast of real-time VRES production in the day-ahead stage to assess the costs incurred for redispatching. ATCs are taken to be non-negative symmetric bounds on the flows $f_{e}$ between zones in the day-ahead market stage. As we consider a single day-ahead market clearing, at most a single bound on $f_{e}$ can be active at a time. Thus, using asymmetric bounds on $f_{e}$ will not improve the results.

We use the term 'real-time market' for any mechanism which modifies the day-ahead dispatch after it is given. The net effect of these mechanisms is to re-dispatch the system to an operation point that is feasible with respect to the full nodal transmission description and actual production of renewable sources. We employ the usual DC flow description for the nodal transmission model, and assume generators are re-dispatched in the cheapest possible manner, that is, the realtime market is fully coordinated between zones.

Each generator $g$ is assumed to offer into the real-time market at a price that differs from its marginal cost (day-ahead offer) by $\pi_{g}^{\uparrow}$ for up-regulation and $\pi_{g}^{\downarrow}$ for down-regulation, such that it is more expensive to re-dispatch a generator closer to real-time. These costs represent in a simplified manner the difference between day-ahead and real-time costs due to reduced system flexibility closer to real-time, or rent-seeking by producers. Premiums for re-dispatch are generally asymmetric: a simple estimate based on comparing day-ahead and intra-day prices for Nord Pool, reveals that down-regulation may actually comes at a higher premium than up-regulation, see Section IV. We do not consider inter-temporal constraints such as ramp limits and storage, or non-convexities in costs associated with unit commitment decisions, assuming that these are represented by the generators through their offer prices. These assumptions make the day-ahead and real-time market clearing problem convex, which is necessary for our formulation. Even though this structure for real-time markets is simplified compared to, e.g., [10] it suffices to examine the impact of endogenous ATC definition.

To evaluate the need for re-dispatch in the real-time market, the ATC optimizer must have a set of scenarios $\Omega$ representing potential realizations of uncertain components, e.g. production of renewable energy sources or consumer demand. While any implementation of a market design which depends on scenarios must define who generates the scenarios and how they are defined, in this work we take the scenarios for given. We consider an in-depth discussion of the definition of these scenarios outside the scope of the present paper, but note that TSOs use such scenarios today to define traditional ATCs.

In this model, evaluating the costs incurred in real-time requires a description of the transmission network and the cost structure per node, i.e. at a plant level. Though current market structures allow access only to aggregate firm-level offers, in a competitive market, these will reflect the firm's plant-level cost structure. In addition, transmission network information is required, but this is not currently shared between TSOs. However, distributed optimization may allow TSOs to keep the amount of data shared to a minimum, while enabling full coordination on cost-optimal ATC determination.

We neglect strategic behavior of market participants in general, and in particular with respect to the updated ATCs, and assume that the ATC optimizer's offer estimates for the day-ahead market are the offers that end up being given in the day-ahead market. Further, we do not model forward and bilateral markets, whose trades would in practice not be known to the ATC optimizer in advance. As with any approach which assumes perfect information, our method depends upon the belief of market parameters, and produces optimal ATCs accordingly.

The next section formulates the ATC-optimizer's problem as an optimization problem, and builds a computationally tractable version by decomposition.

\section{PROPOSED MODEL}

\section{A. Problem Formulation}

As shown in Fig. 1, the ATC optimizer seeks a set of ATCs which minimize the expected cost of operation, when all participants trade in the day-ahead market with those ATCs, and a corrective action happens in real-time. The ATC optimizer must solve its problem under its belief about the day-ahead (DA) and real-time (RT) correction per scenario.

Building on the sketch in (1), we need to explicitly define the day-ahead and real-time market clearing problems. To this end, given a set of ATCs, the zonal network is used to clear the day-ahead market as

$$
\begin{aligned}
& \min _{\Xi \mathrm{DA}} \operatorname{Cost}^{\mathrm{DA}}=\sum_{g} \pi_{g} p_{g}^{\mathrm{DA}} \\
& +\sum_{z} V O L L_{z} l_{z}^{\mathrm{s}, \mathrm{DA}}+\pi^{\mathrm{r}} \sum_{z} w_{z}^{\mathrm{DA}} \\
& \text { s.t. } \quad \sum_{g \in G_{z}} p_{g}^{\mathrm{DA}}+w_{z}^{\mathrm{DA}}+l_{z}^{\mathrm{s} \mathrm{DA}}=L_{z}^{\mathrm{DA}}+ \\
& +\sum_{e \in z \rightarrow} f_{e}^{\mathrm{DA}}-\sum_{e \in \rightarrow z} f_{e}^{\mathrm{DA}} \\
& \forall z \in Z \text { (2b) } \\
& 0 \leq p_{g}^{\mathrm{DA}} \leq P_{g}^{\max } \\
& 0 \leq w_{z}^{\mathrm{DA}} \leq W_{z}^{\mathrm{DA}} \\
& 0 \leq l_{z}^{\mathrm{s}, \mathrm{DA}} \leq L_{z}^{\mathrm{DA}} \\
& \forall z \in Z(2 \mathrm{~d}) \\
& -A T C_{e} \leq f_{e}^{\mathrm{DA}} \leq A T C_{e} \\
& \forall e \in E
\end{aligned}
$$

which yields a day-ahead dispatch $p_{g}^{\mathrm{DA}}$. The variable set $\Xi^{\mathrm{DA}}=\left\{p_{g}^{\mathrm{DA}}, w_{z}^{\mathrm{DA}}, l_{z}^{\mathrm{s}, \mathrm{DA}}, f_{e}^{\mathrm{DA}}\right\}$ includes the day-ahead dispatch per generator, scheduled renewable energy, load shed and flow on each zonal link, respectively.

The objective function ( $2 a)$ minimizes the DA cost, including generation costs of conventional and renewable producers, plus load shedding costs. As renewable energy is treated uniformly across the zone, we simply operate with one renewable production variable per zone. The DA power balance per zone is given by (2b), with subsequent constraints giving bounds on each DA variable. Note that in (2f), $A T C_{e}$ are the ATCs determined in (1). Though they are variables in (1), they are treated as parameters in (2). 
Similarly, the RT redispatch model for each scenario $\omega$ is written as

$$
\begin{aligned}
& \min _{\Xi^{\mathrm{RT}}} \Delta \operatorname{Cost}_{\omega}^{\mathrm{RT}} \\
& =\sum_{g}\left(\pi_{g}\left(p_{g \omega}^{\mathrm{RT}}-p_{g}^{\mathrm{DA}}\right)+\pi_{g}^{\uparrow} p_{g \omega}^{\uparrow}+\pi_{g}^{\downarrow} p_{g \omega}^{\downarrow}\right) \\
& +\sum_{n} V O L L_{n} l_{n \omega}^{\mathrm{s}, \mathrm{RT}}+\pi^{\mathrm{r}} \sum_{n} w_{n \omega}^{\mathrm{RT}} \\
& -\left[\sum_{z} V O L L_{z} l_{z}^{\mathrm{s}, \mathrm{DA}}+\pi^{\mathrm{r}} \sum_{z} w_{z}^{\mathrm{DA}}\right] \\
& \text { s.t. } \quad \sum_{g \in G_{n}} p_{g \omega}^{\mathrm{RT}}+w_{n \omega}^{\mathrm{RT}}+l_{n \omega}^{\mathrm{s}, \mathrm{RT}}=L_{n}^{\mathrm{RT}}+ \\
& +\sum_{l \in n \rightarrow} f_{l \omega}^{\mathrm{RT}}-\sum_{l \in \rightarrow n} f_{l \omega}^{\mathrm{RT}} \\
& p_{g \omega}^{\mathrm{RT}}=p_{g}^{\mathrm{DA}}+p_{g \omega}^{\uparrow}-p_{g \omega}^{\downarrow} \\
& f_{l \omega}^{\mathrm{RT}}=B_{l}\left(\theta_{n \omega}-\theta_{m \omega}\right) \\
& 0 \leq p_{g \omega}^{\mathrm{RT}} \leq P_{g}^{\max } \\
& 0 \leq p_{g \omega}^{\uparrow}, \quad 0 \leq p_{g \omega}^{\downarrow} \\
& 0 \leq w_{n \omega}^{\mathrm{RT}} \leq W_{n \omega}^{\mathrm{RT}} \\
& 0 \leq l_{n \omega}^{\mathrm{s}, \mathrm{RT}} \leq L_{n}^{\mathrm{RT}} \\
& -F_{l}^{\max } \leq f_{l \omega}^{\mathrm{RT}} \leq F_{l}^{\max } \\
& \theta_{n \omega}=0 \\
& \forall(n, m)=l \in L(3 \mathrm{~d}) \\
& \forall n \in N_{\mathrm{Sl}}
\end{aligned}
$$$$
\forall g \in G(3 \mathrm{c})
$$$$
\forall g \in G(3 \mathrm{e})
$$$$
\forall g \in G \text { (3f) }
$$$$
\forall n \in N(3 \mathrm{~g})
$$$$
\forall n \in N(3 \mathrm{~h})
$$$$
\forall l \in L \quad(3 \mathrm{i})
$$

$\forall n \in N$

where $\Xi^{\mathrm{RT}}=\left\{p_{g \omega}^{\mathrm{RT}}, p_{g \omega}^{\uparrow}, p_{g \omega}^{\downarrow}, w_{n \omega}^{\mathrm{RT}}, l_{n \omega}^{\mathrm{s}, \mathrm{RT}}, f_{l \omega}^{\mathrm{RT}}, \theta_{n \omega}\right\}$ contains the real-time generator dispatch, the up- and down-regulation per generator, the delivered renewable production, load shed, flow per transmission line and voltage phase angle.

Objective function (3a) minimizes the RT rebalancing cost, including the regulation costs of generators and load shedding costs. The power balance per node is given by (3b). The coupling of the DA and RT schedules through balancing actions is given by (3c), while transmission flows are given in (3d). Equations (3e)-(3j) define bounds on the RT decision variables and zeros the phase angle of each slack bus. In particular, (3i) restricts the flow on a line to its actual capacity. Note that the upper bound of ( $3 \mathrm{~g})$, i.e., $W_{n \omega}^{\mathrm{RT}}$, is the uncertain parameter which differs from scenario to scenario. The dayahead dispatch variables $p_{g}^{\mathrm{DA}}, l_{z}^{\mathrm{s}, \mathrm{DA}}$ and $w_{z}^{\mathrm{DA}}$ are received from (2), and treated as parameters in the RT problem, (R2.3) ensuring the sequential nature of the day-ahead and real-time markets is preserved.

\section{B. Problem Decomposition}

Both lower-level problems (2) and (3) are linear and thus convex, so their Karush-Kuhn-Tucker (KKT) conditions are optimality conditions. However, a naive implementation of (1) which replaces (2) and (3) by their KKT conditions would result in a computationally intractable problem. In order to allow our method to solve problems of a realistic size, it is necessary to decompose (1) into smaller problems - in this particular problem, we decompose problem (1) by scenario.

For a fixed DA dispatch, the constraints in (1) are independent per scenario. This allows rewriting the problem using Benders' decomposition, where the subproblems are the RT redispatch problems. Taking the complicating (fixing) variables to be $p_{g}^{\mathrm{DA}}$, we write Benders' subproblem at iteration $m$ for scenario $\omega$ as

$$
\begin{aligned}
\min _{\Xi^{\mathrm{RT}}} & \Delta \operatorname{Cost}_{\omega}^{\mathrm{RT}} \\
\text { s.t. } & p_{g}^{\mathrm{DA}}=p_{g}^{\mathrm{DA} ;(m)}: \gamma_{g, \omega}^{(m)} \\
& \Xi^{\mathrm{RT}} \in(3)_{\omega}
\end{aligned}
$$

where the dual variable $\gamma_{g, \omega}^{(m)}$ is the sensitivity to the DA dispatch of generator $g$, used for generating Benders' cuts. The subscript $\omega$ in (4c) indicates the corresponding problem for the given scenario.

As the objective function in (4) is the same as in (3), the bilevel structure of subproblem (4) collapses to

$$
\begin{array}{rlr}
\min _{\Xi^{\mathrm{RT}}} & \Delta \operatorname{Cost}_{\omega}^{\mathrm{RT}} \\
\text { s.t. } & (3 \mathrm{~b})_{\omega}-(3 \mathrm{j})_{\omega} & \\
& p_{g}^{\mathrm{DA}}=p_{g}^{\mathrm{DA} ;(m)}: \gamma_{g, \omega}^{(m)} & \forall g \in G
\end{array}
$$

Unlike (4), subproblem (5) no longer has a bilevel structure. It is now explicitly convex as a function of the DA dispatch, which guarantees that the decomposed problems converge to the optimal solution of the non-decomposed problem (1) [16].

The Benders' master problem in iteration $m$ is

$$
\begin{aligned}
\min _{\Xi \mathrm{DA}, A T C_{e}} & \operatorname{Cost}^{\mathrm{DA}}+\sum_{\omega} \phi_{\omega} \alpha_{\omega} \\
\text { s.t. } & \alpha_{\omega} \geq \alpha^{\min } \quad \forall \omega \in \Omega \\
& \alpha_{\omega} \geq \Delta \operatorname{Cost}_{\omega}^{\mathrm{RT} ;(i)}+\sum_{g} \gamma_{g, \omega}^{(i)}\left(p_{g}^{\mathrm{DA}}-p_{g}^{\mathrm{DA} ;(i)}\right) \\
& \forall i \in\{1, \ldots, m-1\}, \omega \in \Omega \\
& \operatorname{Cost}^{\mathrm{DA}} \in(2) \\
& A T C_{e} \geq 0 \quad \forall e \in E
\end{aligned}
$$

where ${ }^{(i)}$ indicates the variable's fixed value at iteration $i$. The proxy variable $\alpha_{\omega}$ represents the RT redispatch cost under scenario $\omega$. The optimality cuts in (6c) are from Benders' multicut method [17], which was found to yield faster convergence in our applications. As the subproblems (5) are always feasible for any fixed DA dispatch, no feasibility cuts are necessary in (6). The Benders' iterations converge in iteration $m$ if $\sum_{\omega} \phi_{\omega} \alpha_{\omega}^{(m)}=\sum_{\omega} \phi_{\omega} \Delta \operatorname{Cost}_{\omega}^{\mathrm{RT} ;(m)}$ to within tolerance.

Note that Benders' master problem (6) still has a bilevel structure. It is transformed by replacing the lower-level optimization problem (6d) by its KKT conditions, and reformulating these using the standard Big-M method [18], yielding a mixed-integer linear programming problem which we omit here for brevity.

Two modifications are made to (5) and (6) to reduce complexity and handle degeneracy. First, to reduce the number of variables exchanged between master and subproblems, we redefine for each scenario $\omega$

$$
\begin{aligned}
\Delta \operatorname{Cost}_{\omega}^{\mathrm{RT}}= & \sum_{g}\left(\pi_{g}\left(p_{g \omega}^{\mathrm{RT}}-p_{g}^{\mathrm{DA}}\right)+\pi_{g}^{\uparrow} p_{g \omega}^{\uparrow}+\pi_{g}^{\downarrow} p_{g \omega}^{\downarrow}\right) \\
& +\sum_{n} V O L L_{n} l_{n}^{\mathrm{s}, R T}+\pi^{\mathrm{r}} \sum_{n} w_{n \omega}^{\mathrm{RT}},
\end{aligned}
$$



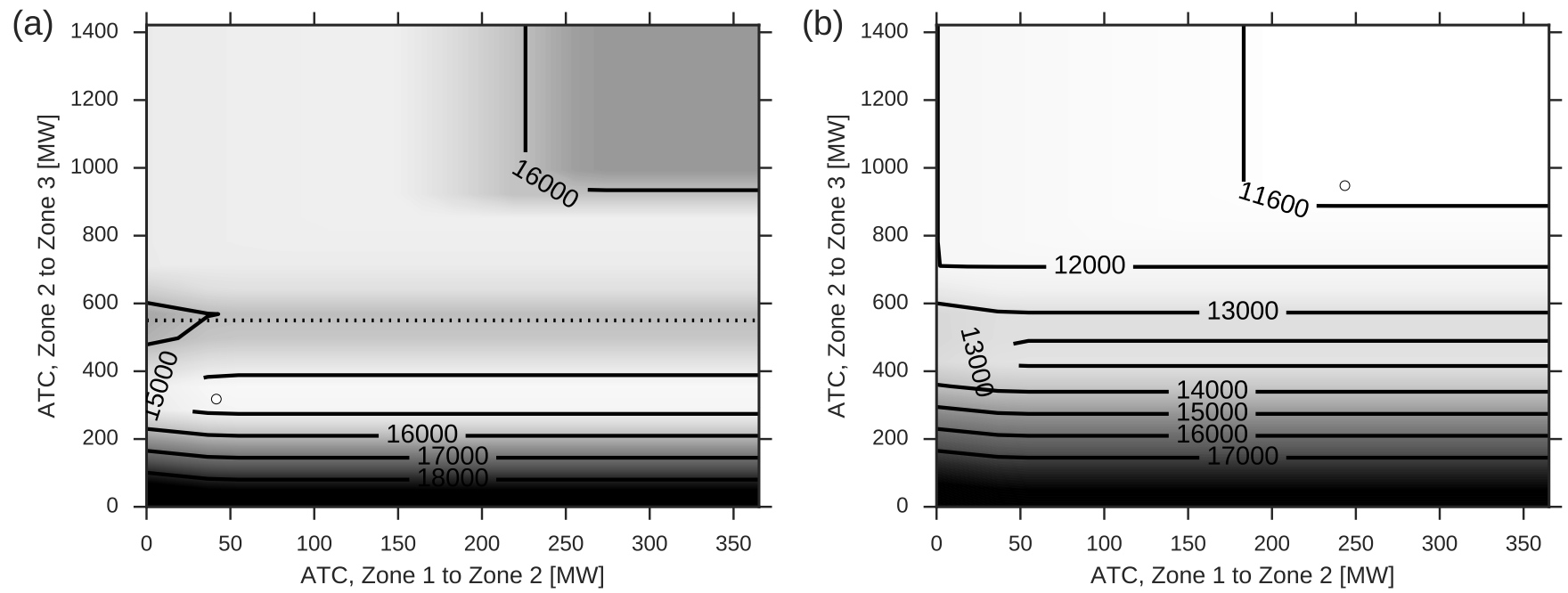

Fig. 3. The 24-bus IEEE reliability test system: Final expected cost [\$] as function of ATCs with the capacity on the intrazonal line 15-24 set to (a) 150 MW, (b) $500 \mathrm{MW}$. The circle indicates the cost-optimal ATCs found by the proposed formulation. The dotted line in (a) indicates a region of high cost separating two regions of lower cost.

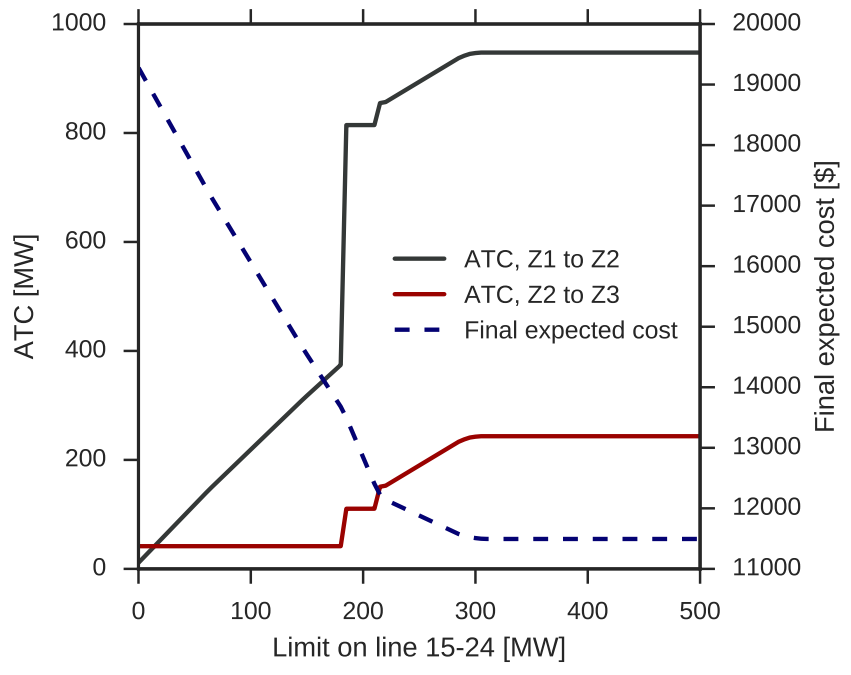

Fig. 2. The 24-bus IEEE reliability test system: Cost-optimal ATCs found by the proposed formulation (left axis) and final expected cost (right axis) as a function of the capacity on the intrazonal line $15-24$.

which is equivalent to (3a) with the term in square brackets removed. We move this term to Benders' cuts, (6c), which now writes as

$$
\begin{aligned}
& \alpha_{\omega} \geq \Delta \operatorname{Cost}_{\omega}^{\mathrm{RT} ;(i)}+\left[\sum_{z} \operatorname{VOLL}_{z} l_{z}^{\mathrm{s}, \mathrm{DA} ;(i)}+\pi^{\mathrm{r}} \sum_{z} w_{z}^{\mathrm{DA} ;(i)}\right] \\
& +\sum_{g} \gamma_{g, \omega}^{(i)}\left(p_{g}^{\mathrm{DA}}-p_{g}^{\mathrm{DA} ;(i)}\right) \quad \forall i \in\{1, \ldots, m-1\}, \omega \in \Omega
\end{aligned}
$$

As a result, only the DA schedule of conventional generators $\left(p_{g}^{\mathrm{DA}}\right)$ needs to be shared between master and subproblems.

Second, in solving the above problem, it may happen that some ATC constraints are not binding. This leads to an unbounded ray, where the cost does not change when the ATC goes to infinity. To avoid this, we add the penalty term
TABLE I

ZONES FOR IEEE RELIABILITY TEST SYSTEM

\begin{tabular}{llll} 
& Zone 1 & Zone 2 & Zone 3 \\
\hline Nodes in zone & $1,2,4-6$ & $3,7-14$ & $15-24$
\end{tabular}

$\epsilon \sum_{e} A T C_{e}$ to (6a), where $\epsilon$ is a small value, e.g., $10^{-6}$. This term ensures that among possible ATCs which lead to a degenerate cost, the smallest ATC is chosen. We proceed to apply this final formulation to a series of test cases.

\section{CAse Studies}

To show the results of optimizing ATCs, we employ two test cases. By first calculating the optimal set of ATCs for a small test system, we show that the method is sensitive to having access to the precise information on the network. The second test system shows how our method scales to largescale systems, and examines the impact of employing optimal ATCs on the expected total cost of operation. This second test system further demonstrates a lower bound on the efficiency of European zonal markets.

The optimization problems (5) and (6) were implemented in Python using the Gurobi solver package. This implementation solves the large-scale test case to a $1 \%$ Benders gap in 14 hours. All codes used are available at [19], where a brief overview of the solution strategy is also available.

\section{A. Test Case: IEEE 24-bus Reliability Test System}

To verify the implementation and show some interesting features of the method, we use a modified version of the IEEE 24-bus reliability test system [20]. This system is divided into the three zones listed in Table I, and connected via two interconnections (links); one from zone 1 to zone 2, and one from zone 2 to zone 3 . The low dimensionality of this system allows directly plotting final expected cost as a function of the 

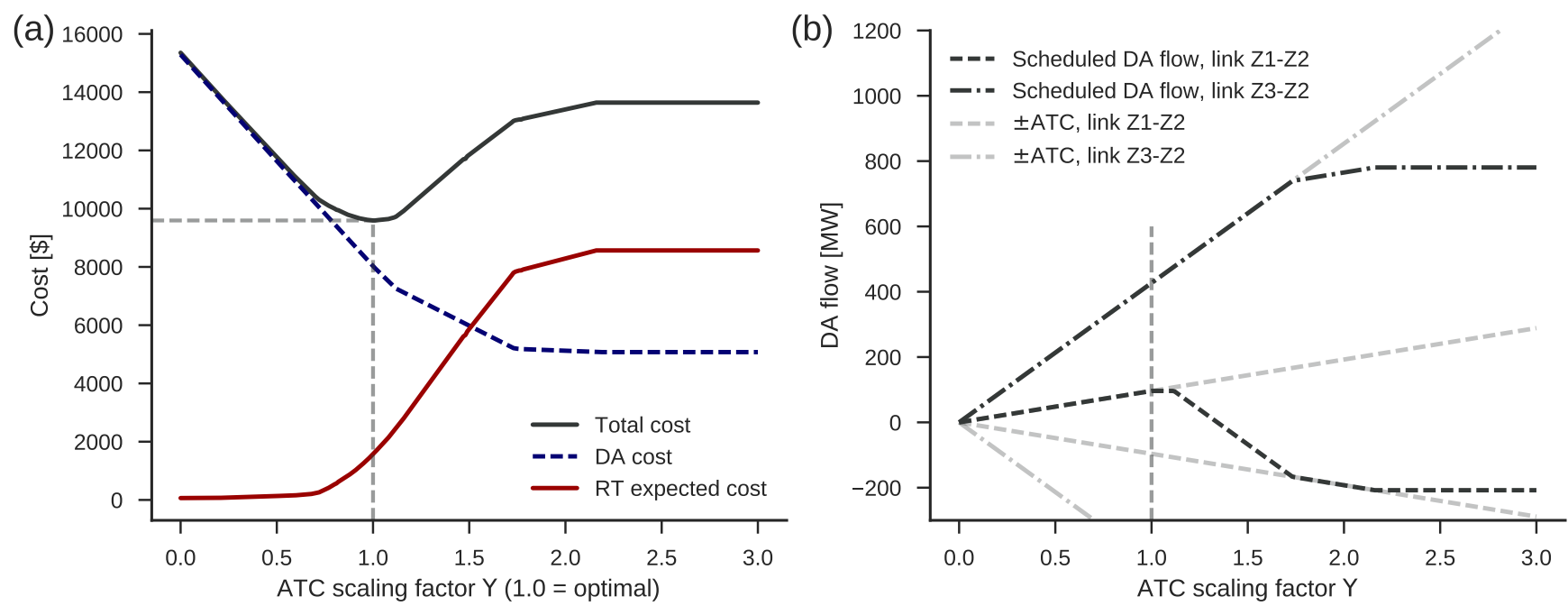

Fig. 4. The 24-bus IEEE reliability test system: Resulting costs (a) and flows (b) from a unilateral rescaling of the set of optimal ATCs by $\Upsilon$.

ATCs on each link. For this example, we examine the costoptimal ATCs for the first hour.

The modifications to the base system [20] are as follows: $50 \mathrm{MW}$ of wind capacity is placed at each of nodes 3,5 , 7, 16, 21 and 23. Each wind generators' output per scenario is drawn independently from a Beta distribution with shape parameters $\alpha=2$ and $\beta=4$. In total, 100 scenarios for wind generation are used to schedule the system. We assume redispatch premiums are $\pi_{g}^{\uparrow}=\$ 7.90 / \mathrm{MWh}$ and $\pi_{g}^{\downarrow}=\$ 8.59 / \mathrm{MW} \mathrm{h}$ independent of the generator. By varying the capacity limit on the line connecting nodes 15 and 24 , both of which are inside zone 3 , we show the sensitivity of our method to network conditions. Fig. 2 shows the costoptimal ATCs as a function of the thermal limit on line 1524 , together with the resulting final expected cost. The costoptimal ATCs change drastically for capacity limits around $200 \mathrm{MW}$, indicating that they depend non-trivially on actual network characteristics.

This is further indicated by Fig. 3, which shows final expected cost as a function of ATCs for two different capacities on the line connecting nodes 15 and 24. For a line capacity below $200 \mathrm{MW}$, the optimal ATCs are in the lower left corner of Fig. 3 (a). In contrast, for a line capacity well above $200 \mathrm{MW}$, the optimal set of ATCs are in the upper right corner of Fig. 3 (b). Between these two points there is a barrier of higher cost, indicated by the dotted line in Fig. 3 (a) at approximately $500 \mathrm{MW}$ on the vertical axis. As the line capacity increases from below $200 \mathrm{MW}$ to above, the optimal set of ATCs must cross this barrier, leading to the sudden change observed in Fig. 2. The existence of this barrier shows that final expected cost is in general a non-convex function of $A T C_{e}$.

This non-convexity of costs and cost-optimal ATCs indicates that our method of ATC determination is highly sensitive to the input data used to generate ATCs. Such sensitivity poses problems for any potential market design based on deriving cost-optimal ATCs, as small errors in the market information may lead to disproportionate errors in operational costs. This may be partially addressed by extending the method to optimize against scenarios of market conditions, e.g., offer price of conventional generators, in addition to wind scenarios.

Additionally, to illustrate the connection between ATC strength and system cost in DA versus expected system cost in RT, we set the capacity on line 15-24 to $150 \mathrm{MW}$, and upscale the nameplate capacity of each turbine to $300 \mathrm{MW}$. For this configuration, we find the set of cost-optimal ATCs. Then, we scale these ATCs by a factor $\Upsilon \in[0,3]$, and evaluate the costs incurred by using these exogenous ATCs. The resulting costs are graphed in Figure 4 (a). As $\Upsilon$ is decreased below 1.0, DA system cost increases as zones cannot share the lowest-cost resources between them. However, there is not a corresponding decrease in expected real-time cost. In this example, once each zone is self-sufficient, re-scaling takes place between units with degenerate costs, leading to no change in expected cost.

On the other hand, when $\Upsilon$ is increased above 1.0, the dayahead market schedules more energy to be shared between zones. However, once real-time uncertainty reveals itself, the flows induced by this high sharing are found to be incompatible with constraints on the underlying grid, leading to a high need for re-balancing and subsequent high real-time cost. This is further evident by the flow on the link $Z 1 \rightarrow Z 2$ changing direction for very high ATCs, see Figure 4 (b). The optimal set of ATCs, where $\Upsilon=1.0$, represents a compromise between these two contributions.

\section{B. Test Case: European-Scale System Model}

To examine the limitations of zonal pricing, we examine our model applied to the RE-Europe test system [21]. This test system contains 1500 nodes, 2000 lines and 969 generators, and comes with thermal limits for inter-country lines, wind and solar point forecasts and signals at hourly resolution as well as hourly load signals. We use the 'uniform' capacity layout for wind and solar power, with a base scenario of 50\% gross VRES penetration, of which $80 \%$ of yearly energy production 

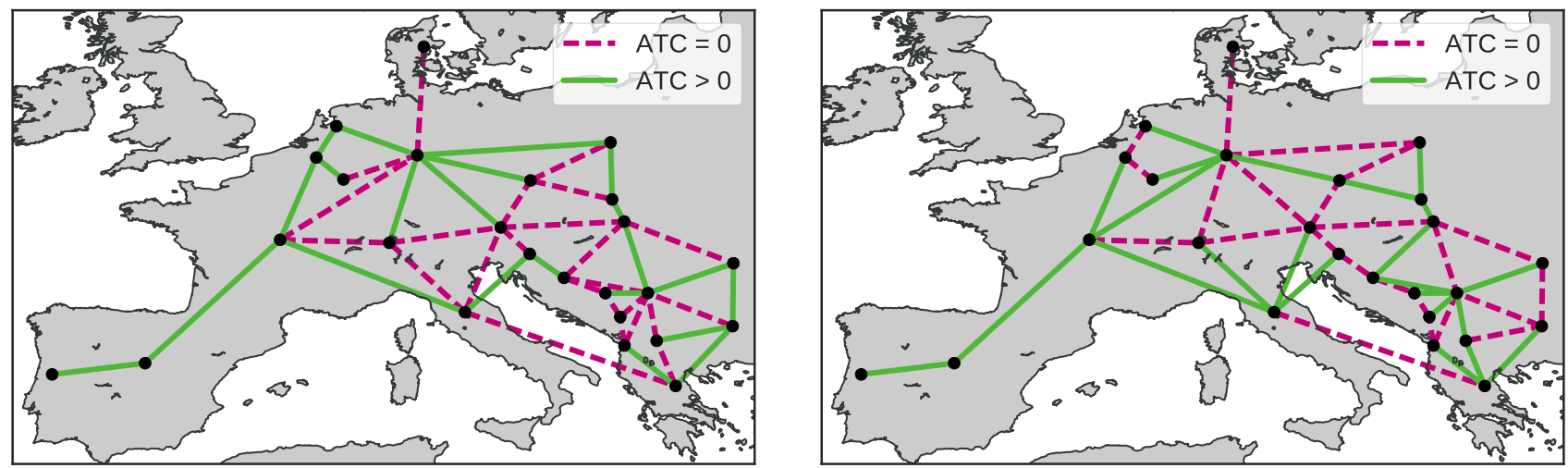

Fig. 6. European-scale system model: Qualitative map of cost-optimal ATCs found for (left) hour 17 and (right) hour 18 .

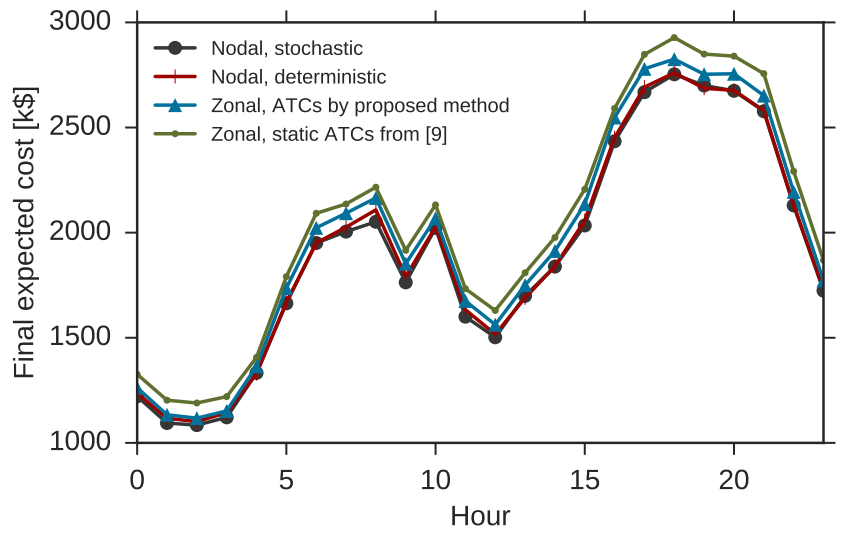

Fig. 5. European-scale system model: Final expected costs per hour.

comes from wind and $20 \%$ from solar. We generate scenarios from the supplied point forecasts from ECMWF data, with the real-time signal derived from the COSMO dataset, for details, see [21]. These scenarios have the correct empirical spatial dependence structure, while being uncoupled in time, which is of no importance in our formulation, see [19] for more details on scenario generation.

Each country in the test system is assumed to comprise a single market zone, and two zones are connected if they have a transmission line connecting them in the underlying grid. The resulting zonal configuration comprises 25 zones with 46 interconnections between them. These assumptions neglect the division of Italy and Denmark into separate bidding zones, anticipate the splitting of Austria and Germany into two separate zones as recommended in [22], and assumes all countries have national electricity markets, even though some do not.

Due to the size of the test system, we divide the problem by hours and solve for a cost-optimal ATC for each hour separately. This is in line with current practice in, e.g., Nordpool, where ATCs are updated hour by hour [23]. We arbitrarily choose to run simulations for each hour of the 15th of June 2014. As before, we assume redispatch premiums are
TABLE II

EUROPEAN-SCALE SYSTEM MODEL: MEAN HOURLY COST BY METHOD

\begin{tabular}{lccc} 
& $\begin{array}{c}\text { DA Cost } \\
{[\mathrm{k} \$]}\end{array}$ & $\begin{array}{c}\text { Final expected } \\
\text { cost }[\mathrm{k} \$]\end{array}$ & $\begin{array}{c}\text { \% increase } \\
\text { in final cost }\end{array}$ \\
\hline Nodal, Stochastic (baseline) & 3915 & 1902 & - \\
Nodal, Deterministic & 1559 & 1917 & 0.7 \\
Zonal, Cost-optimal ATCs & 1655 & 1970 & 3.6 \\
Zonal, static ATCs from [9] & 1929 & 2040 & 7.3
\end{tabular}

$\pi_{g}^{\uparrow}=\$ 7.90 / \mathrm{MWh}$ and $\pi_{g}^{\downarrow}=\$ 8.59 / \mathrm{MWh}$ independent of the generator. These values are the median-of-median differences between day-ahead and regulating prices in Nordpool for 2014 [23]. Our results are insensitive to an overall shift of these premiums, up to their trivial effect on redispatch costs. The specifics of our results may change if the premiums differ per generator, but we consider a full treatment of this effect outside the scope of this paper.

We compare our cost-optimized ATCs against (i) a stochastic nodal dispatch in which the nodal network is used in both DA and RT stages considering the full set of scenarios, (ii) a deterministic nodal dispatch in which the uncertainty is ignored - the expected renewable production is considered as the single scenario, and (iii) a deterministic zonal dispatch using static ATCs derived as in [9]. Considering 10 scenarios, our proposed method generated cost-optimal ATCs, and the stochastic nodal model found an optimal DA schedule. The resulting DA schedule and ATCs were then evaluated using 90 scenarios different from the initial 10. Renewables are assumed to offer their mean production under the applicable scenarios into the DA market.

The resulting final expected costs are shown in Fig. 5, which shows that our method achieves an operational costs between the static ATCs and both nodal formulations for all hours considered. The mean of final expected cost over the horizon is given in Table II. The cost difference between the stochastic and deterministic nodal models $(0.7 \%)$ relates to the gain in efficiency from considering uncertainty directly. As the gap in final expected cost between the proposed formulation and the static ATCs is greater at 3.5\%, our method achieves gains greater than would be expected from just considering VRES uncertainty. The use of static ATCs overestimates the 
TABLE III

EUROPEAN-SCALE SYSTEM MODEL: UP- AND DOWN-REGULATION RELATIVE TO FINAL PRODUCTION

\begin{tabular}{lcc} 
& $\begin{array}{c}\text { Mean up- } \\
\text { regulation [\%] }\end{array}$ & $\begin{array}{c}\text { Mean down - } \\
\text { regulation [\%] }\end{array}$ \\
\hline Nodal, Stochastic (baseline) & 7.01 & 6.38 \\
Nodal, Deterministic & 9.74 & 4.87 \\
Zonal, Cost-optimal ATCs & 10.66 & 6.96 \\
Zonal, static ATCs from [9] & 12.19 & 9.58
\end{tabular}

TABLE IV

EUROPEAN-SCALE SYSTEM MODEL: EXAMPLE ATCS IN MW OBTAINED BY THE PROPOSED MODEL, THE METHOD OF [9] AND THE TOTAL CROSS-BORDER CAPACITY OF THE UNDERLYING GRID.

\begin{tabular}{lrrrr} 
& \multicolumn{2}{c}{ Cost-optimal ATC } & Static ATC & Cross-border \\
Link & hour 17 & hour 18 & from [9] & capacity \\
\hline GER-FRA & 0 & 10139 & 659 & 5130 \\
ITA-FRA & 13510 & 295 & 409 & 4880 \\
ITA-SVN & 2484 & 422 & 414 & 1949
\end{tabular}

efficiency gap between zonal and nodal markets by a factor of roughly 2 . We note, that the number of scenarios used here for the stochastic nodal and cost-optimal dispatches may be insufficient to ensure out-of-sample stability. Adding additional scenarios is expected to improve the operational costs found for these methods relative to their deterministic counterparts, but is unlikely to affect the conclusions drawn.

Table III lists the relative up- and down-regulation, calculated as $\sum_{g} p_{g \omega}^{\uparrow / \downarrow} / \sum_{g} p_{g \omega}^{\mathrm{RT}}$, averaged over scenarios $\omega$ and hours. Using cost-optimal ATCs brings the up- and downregulation required close to the result for the deterministic nodal setup. The difference in up- and down-regulation capacity is due to the solar production forecast overestimating available solar.

However, this gain in efficiency comes with fundamentally different ATCs from today. Fig. 6 qualitatively shows the obtained cost-optimal ATCs for hours 17 and 18. Certain links receive an ATC of zero, preventing trades between the corresponding zones, with the closed-off links changing over time. This binary behavior extends to all hours considered, indicating that it is an inevitable part of defining cost-optimal ATCs. To examine the values in detail, a selection of optimal ATCs are tabulated in Table IV. The GER-FRA link is closed for hour 17, but allows trades up to nearly twice the underlying grid strength in hour 18. In comparison, the ITA-FRA link displays the opposite trend; while initially open to trade for 2.5 times the underlying grid capacity, it reduces in hour 18 to less than the static ATC assignment. The cost-optimal ATC sizes do not always exhibit these extremes as shown by the ITA-SVN link, which fluctuates between 1.2 times the underlying grid strength and the static ATC assignment. The binary behavior shown in Fig. 6 and the fluctuations in Table IV are due to the ATCs being used as indirect control of the dispatch. By constricting ATCs on certain links, zonal prices bring the DA dispatch closer to the ideal of the stochastic nodal model.

\section{CONCLUSIONS}

We propose a method for defining cost-optimal ATCs, whereby an ATC optimizer finds the set of ATCs which lead to the lowest final expected cost under scenarios of renewable production. This separates financial ATCs, as used in electricity markets, from physical ATCs, which are defined based on measures of security. This new notion allows for increasing the efficiency of zonal electricity markets by finding the optimal boundaries for trades among zones from a market perspective. While this view is incongruent with the current market structure, we see cost-optimal ATCs as a way to probe the fundamental limitations of zonal markets, i.e., as an input to the debate on whether there is a way forward for unbundled zonally priced markets in a highly renewable context.

We applied our method to two test cases: an illustrative test case and a European-scale test case. Analysis of the illustrative test case demonstrated that the problem of finding cost-optimal ATCs for a given network and a given set of scenarios is in general a non-convex problem, which may be highly sensitive to available capacities on the underlying network. This sensitivity cautions against naively using costoptimal ATCs in market designs.

Examining a European-scale test system found that our method reduced final expected cost on a daily horizon by $3.5 \%$ compared to a static ATC assignment. Compared to the result of a full nodal model with and without stochastic DA market clearing, final expected costs increase by $3.6 \%$ and $2.9 \%$ respectively. The cost-optimal ATCs found are radically different than those corresponding to current practice, with many connections being closed for trades in one hour, but open for trades in the next. Due to the nature of the test case, the limited time span examined, and the simplified method used to generate the scenarios, one should be careful not to take these numbers as generally representative of the performance gains possible using our method. The numbers do, however, show that the method used to define ATCs may impact the perceived efficiency of zonal market clearing, inviting caution when non-integrated ATCs are used to make strong claims about the efficiency of zonal markets.

To further examine the limitations of zonal markets, the method employed here may be extended to make the definition of the market areas endogenously. Allowing dynamic zoning can only serve to further decrease costs, as using a single fixed topology provides an upper bound to this extension. Further, from a practical standpoint, though cost-optimal ATCs optimize for the highest overall social welfare, some zones may incur a welfare loss. To counteract this, cost-optimal ATCs may be extended to ensure no zone incurs a loss of social welfare from the defined ATCs, either directly or through an uplift system. Such an extension would naturally encompass partial coordination between TSOs on ATC definition. Further extensions of interest are extending the ATC optimizers problem to allocate reserves(R2.4) or handle contingencies and forced outages, the impact of intertemporal constraints such as ramping, and to reconcile the conceptual gap between nonintegrated and cost-optimal ATCs.

\section{ACKNOWLEDGMENT}

The authors would like to thank Stefanos Delikaraoglou (Technical University of Denmark) and Brent Eldridge (Johns 
Hopkins University) for enlightening discussions, and Christos Ordoudis (Technical University of Denmark) for his comments on the final manuscript.

\section{REFERENCES}

[1] J. M. Morales, M. Zugno, S. Pineda, and P. Pinson, "Electricity market clearing with improved scheduling of stochastic production," Eur. J. Oper. Res., vol. 235, no. 3, pp. 765-774, Jun. 2014.

[2] P. Pinson, "Wind energy: Forecasting challenges for its operational management," Stat. Sci., vol. 28, no. 4, pp. 564-585, 2013.

[3] ACER, "The influence of existing bidding zones on electricity markets," vol. 386, no. 34114458, pp. 1-16, 2013.

[4] F. Bouffard, F. D. Galiana, and A. J. Conejo, "Market-clearing with stochastic security - Part I: Formulation," IEEE Trans. Power Syst., vol. 20 , no. 4 , pp. $1818-1826,2005$.

[5] J. M. Morales, A. J. Conejo, and K. Liu, "Pricing electricity in pools with wind producers," IEEE Trans. Power Syst., vol. 27, no. 3, pp. 1366 1376, 2012.

[6] K. Van den Bergh, J. Boury, and E. Delarue, "The flow-based market coupling in central western Europe: Concepts and definitions," Electr. J., vol. 29, no. 1, pp. 24-29, Jan. 2016.

[7] Y. Ou and C. Singh, "Assessment of available transfer capability and margins," IEEE Trans. Power Syst., vol. 17, no. 2, pp. 463-468, May 2002.

[8] L. Min and A. Abur, "Total transfer capability computation for multiarea power systems," IEEE Trans. Power Syst., vol. 21, no. 3, pp. 11411147, 2006.

[9] G. Oggioni, F. Murphy, and Y. Smeers, "Evaluating the impacts of priority dispatch in the European electricity market," Energy Econ., vol. 42, pp. 183-200, Mar. 2014.

[10] I. Aravena and A. Papavasiliou, "Renewable energy integration in zonal markets," IEEE Trans. Power Syst., vol. 32, no. 2, pp. 1334-1349, 2017.

[11] J. Egerer, J. Weibezahn, and H. Hermann, "Two price zones for the German electricity market - Market implications and distributional effects," Energy Econ., vol. 59, pp. 365--381, 2016.

[12] M. Bjorndal and K. Jornsten, "Zonal pricing in a deregulated electricity market," Energy J., vol. 22, no. 1, pp. 1-26, Jan. 2001.

[13] The European Commission, EU Energy Markets in 2014, 2014. [Online]. Available: http://ec.europa.eu/energy/sites/ener/files/ documents/2014_energy_market_en.pdf

[14] J. Dijk and B. Willems, "The effect of counter-trading on competition in electricity markets," Energy Policy, vol. 39, no. 3, pp. 1764-1773, 2011.

[15] S. A. Gabriel, A. J. Conejo, J. D. Fuller, B. F. Hobbs, and C. Ruiz, Complementarity Modeling in Energy Markets, ser. International Series in Operations Research \& Management Science. New York, NY: Springer, 2013.

[16] M. Bagajewicz and V. Manousiouthakis, "On the generalized Benders decomposition," Comput. Chem. Eng., vol. 15, no. 10, pp. 691-700, Oct. 1991.

[17] J. R. Birge and F. V. Louveaux, "A multicut algorithm for two-stage stochastic linear programs," Eur. J. Oper. Res., vol. 34, no. 3, pp. 384 392, Mar. 1988.

[18] J. Fortuny-Amat and B. McCarl, "A representation and economic interpretation of a two-level programming problem," J. Oper. Res. Soc., vol. 32, no. 9, pp. 783-792, Sep. 1981.

[19] T. V. Jensen, J. Kazempour, and P. Pinson, "Cost-optimal ATC code," 2016. [Online]. Available: https://github.com/TueVJ/Bilevel-ATC

[20] C. Grigg and P. Wong, The IEEE reliability test system -1996 a report prepared by the reliability test system task force of the application of probability methods subcommittee. Boston, MA: IEEE, Nov. 1999, vol. 14, no. 3 .

[21] T. V. Jensen, P. Pinson, M. Greiner, and H. de Sevin, "The RE-Europe data set," 2017. [Online]. Available: https://zenodo.org/record/803266

[22] ACER, "ACER opinion 09-2015 on the compliance of NRAs' decisions approving methods of cross-border capacity allocation in the CEE region," Tech. Rep., 2015.

[23] Nord Pool, "Nord pool spot: Market data," 2016. [Online]. Available: www.nordpoolspot.com

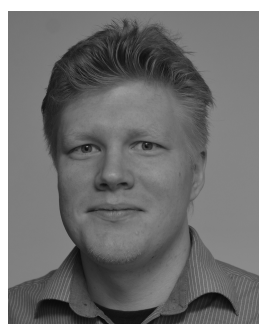

Tue Vissing Jensen (S'14) received the M.Sc. degree in physics from Aarhus University (Denmark) and the Ph.D. degree in electricity markets from the Technical University of Denmark (Denmark). He is currently a postdoctoral researcher at the Technical University of Denmark Center for Electric Power and Energy, Department of Electrical Engineering. His research interests include electricity markets, complex networks, mechanism design and bounded rationality.

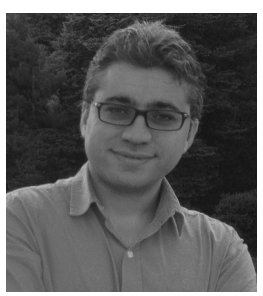

Jalal Kazempour (M'14) is an Assistant Professor at the Department of Electrical Engineering, Technical University of Denmark, Kgs. Lyngby, Denmark. He received his Ph.D. degree in Electrical Engineering from University of Castilla-La Mancha, Ciudad Real, Spain, in 2013. He was a postdoctoral fellow at The Johns Hopkins University, MD, USA, in 2014, and at the Technical University of Denmark in 20152016. His research interests include power systems, electricity markets, optimization and its applications to energy systems.

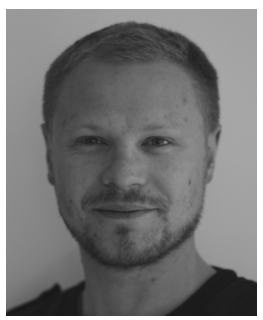

Pierre Pinson (M'11-SM'13) received the M.Sc. degree in applied mathematics from the National Institute for Applied Sciences (INSA Toulouse, France) and the Ph.D. degree in energetics from Ecole des Mines de Paris (France). He is a Professor at the Technical University of Denmark (DTU), Centre for Electric Power and Energy, Department of Electrical Engineering, also heading a group focusing on Energy Analytics \& Markets. His research interests include among others forecasting, uncertainty estimation, optimization under uncertainty, decision sciences, and renewable energies.

Prof. Pinson acts as an Editor for the International Journal of Forecasting, and for Wind Energy. 